\title{
Texte und Kommunikationsangebote der Institution Theater im physischen und virtuellen Raum
}

\author{
Texts of the institution "theatre" in physical and virtual communication space
}

\author{
Tanja ŠKERLAVAJ \\ Justus-Liebig-Universität Gießen/ \\ E-Mail: Tanja.Skerlavaj@germanistik.uni-giessen.de,
}

\begin{abstract}
In the external communication of theatres there is a number of „communicative tasks” (T. Luckmann 1986) that have to be completed. In regard to these tasks, a broader communicative structure of texttypes and genres has developed, which can be referred to as a „communicative ecology”. At the beginning of the paper this complex structure is briefly presented. Subsequently, the paper deals with two important components of the ecology in the external communication of theatres: the physical and the virtual communication space. The objective of the paper is to describe the typical characteristics of placebound texts in the physical space and texts in the digital space of the cultural institution ,theatre". In this way it should be explored how (that is, on the basis of which linguistic elements and other semiotic resources) and for what purposes the institution „theatre” communicates through its place-bound and digital texts with its (potential) customers. As texts in such ,genre networks” (K. Adamzik 2011) are related to one another, the paper also aims to determine the intertextual relations between the analysed texts in both communication spaces.
\end{abstract}

Keywords: Text linguistics, communicative ecology, genre networks, physical and digital communication space, place-bound texts, digital texts, multimodality, intertextual relations

\section{Einleitung}

In Kunstinstitutionen wie etwa Schauspielhäusern kommt Texten eine zentrale Rolle zu. Dabei spielen in erster Linie literarische Texte und Bühnendarbietungen eine besonders wichtige Rolle, es sollen jedoch auch andere Texte und Kommunikationsangebote nicht vernachlässigt werden - z. B. solche, durch welche Theaterinstitutionen intern oder nach außen kommunizieren. So sind beispielsweise in der Außenkommunikation von Theatern eine ganze Reihe typischer und wiederkehrender kommunikativer Aufgaben (T. Luckmann 1986) zu erfüllen, in Bezug auf welche sich evolutionär ein eigenes Kommunikationsaufkommen herausgebildet hat, das man als eine „Ökologie" von Textsorten und Arten von multimodalen Angeboten bezeichnen kann"

${ }^{1}$ Diese Thematik ist in ein größeres Forschungsprojekt eingelagert, das gleichzeitig als Habilitationsvorhaben dient. Darin sollen die einzelnen Textsorten in der Außenkommunikation von Theatern untersucht werden, außerdem soll der Frage nachgegangen werden, welche Arten von Zusammenhängen es zwischen den unterschiedlichen Texttypen gibt und wie sich die innere Struktur dieses „Textsortenkosmos“ funktional charakterisieren lässt. 
Im vorliegenden Beitrag soll zunächst die „kommunikative Ökologie“ der Institution Theater kurz geschildert werden (Abschnitt 2). Anschließend wendet sich der Beitrag zwei wichtigen Komponenten der Textökologie in der Außenkommunikation von Theatern zu: dem physischen und dem virtuellen Kommunikationsraum (und innerhalb des physischen Raums insbesondere den ortsgebundenen bzw. „meso-kommunikativen“ Texten - vgl. C. Domke 2014; Abschnitt 3). Damit werden im Beitrag zwei Ziele verfolgt: Zum einen sollen die charakteristischen Merkmale der ortsgebundenen bzw. meso-kommunikativen Texte im physischen Raum und der Texte im digitalen Raum der Theaterinstitutionen bestimmt und gründlich beschrieben werden. Dadurch soll festgestellt werden, wie (d.h. z. B. mit welchen sprachlichen Mitteln und anderen semiotischen Ressourcen) und zu welchen Zwecken die Institution Theater anhand ihrer ortsgebundenen und digitalen Texte mit ihrem (potenziellen) Publikum kommuniziert sowie welche Themen dabei mitverhandelt werden. Da dem Kernbereich dieser kommunikativen Ökologie angehörende Texte durch ein sogenanntes „Textsortennetz“(K. Adamzik 2011) dargestellt werden können und anhand verschiedener Relationen miteinander verbunden sind, soll im Beitrag zum anderen erforscht werden, ob die digitalen Kommunikationsangebote bestimmte Eigenschaften der „lokalen" Textformen aufweisen bzw. ob zwischen den Texten im physischen und virtuellen Raum der Institution Theater gewisse Zusammenhänge bestehen.

Diesen Fragen soll nachgegangen werden, indem ,physische“ und digitale Texte bzw. kommunikative Angebote aus drei verschiedenen Theaterinstitutionen aus textlinguistischer und multimodaler Perspektive, d.h. im Hinblick auf ihre grundlegenden Funktionen und sprachlichen Handlungen, ihre (Teil-)Themen, Formulierungsmuster, Textträger und Multimodalität, exemplarisch analysiert werden (Abschnitte 3.1 und 3.2) und schließlich hinsichtlich der Relationen zwischen ihnen geprüft werden (Abschnitt 4).

\section{Die Ökologie in der Außenkommunikaton von Theatern}

In der Außenkommunikation von Theatern sind verschiedene kommunikative Aufgaben zu erfüllen, die mit der Produktion, Vermittlung und Vermarktung performativer Kunst im Kontext von gedruckten (Massen-)Medien bzw. Netzöffentlichkeit zusammenhängen. In Bezug auf diese Aufgaben wie etwa ,über das Programm informieren“, „für Aufführungen werben“, „Abonnements anbieten”, „die Theaterinstitution und ihre Mitarbeiter vorstellen" usw. hat sich eine sog. „Ökologie” von Textsorten und multimodalen Angeboten ${ }^{2}$ herausgebildet, deren Kernbereich durch ein „Textsortennetz“ (K. Adamzik 2011) dargestellt werden kann. Dazu gehören die folgenden Textsorten und multimodalen Angebote: Spielzeitheft, Spielplan, Website, Schauspielerporträt, Abo-Heft, Programmheft, Vorankündigung, Newsletter, Facebook-

\footnotetext{
${ }^{2}$ Die Idee einer Kommunikations-Ökologie führt auf frühere Konzeptionen zur Beschreibung solcher komplexen Funktionszusammenhänge zurück, wie etwa die des ,kommunikativen Haushalts“ (T. Luckmann 1986, 1997), der „Textsorten-Intertextualität“ (J. Klein 1991, 2000) und der „Textsortennetze“ (K. Adamzik 2000, 2011 sowie N. Janich 2009). Zu einer ausführlicheren Auseinandersetzung mit diesen Konzeptionen siehe T. Škerlavaj 2018.
} 
Profilseite und Twitter-Seite, Werbetext, Stückzettel, Theaterkritik und „empraktische" Kommunikationsangebote ${ }^{3}$ wie etwa Eintrittskarten oder verschiedene Schilder und Wegweiser. Die diesem Netz angehörenden Textsorten und multimodalen Angebote weisen eine große Vielfalt in Form, Thema und Funktion auf und sind aufgrund verschiedener (z. B. thematischer, funktionaler, formaler, zeitlicher, medialer, situativer usw.) Relationen miteinander verbunden. Dabei folgen manche kommunikativen Angebote traditionellen Darstellungsmustern, es finden sich im Umkreis von Theatern aber nicht selten auch innovative Realisierungsvarianten, deren Zusammenspiel mit zum Gegenstand der Untersuchung gehört.

$\mathrm{Zu}$ dieser komplexen Ökologie in der Außenkommunikation von Theatern gehören zudem weitere Komponenten wie etwa die an dieser Kommunikation beteiligten Akteure, die typische zeitliche Dynamik der kommunikativen Angebote in einer Spielzeit, die Nutzung von Medien, durch welche die Angebote vermittelt werden, und die Frage der räumlichen Prägung (,physisch“ vs. „,virtuell“), sodann verschiedene Aspekte der Lokalität (,ortsgebunden“ vs. „ortsungebunden“) usw.

Diesen komplexen Funktionskreis zwischen Angebotsrepertoire (Textsortennetz), Akteuren, der typischen zeitlichen Dynamik, der Ort- und Mediennutzung sowie der räumlichen Prägung veranschaulicht die folgende Grafik:

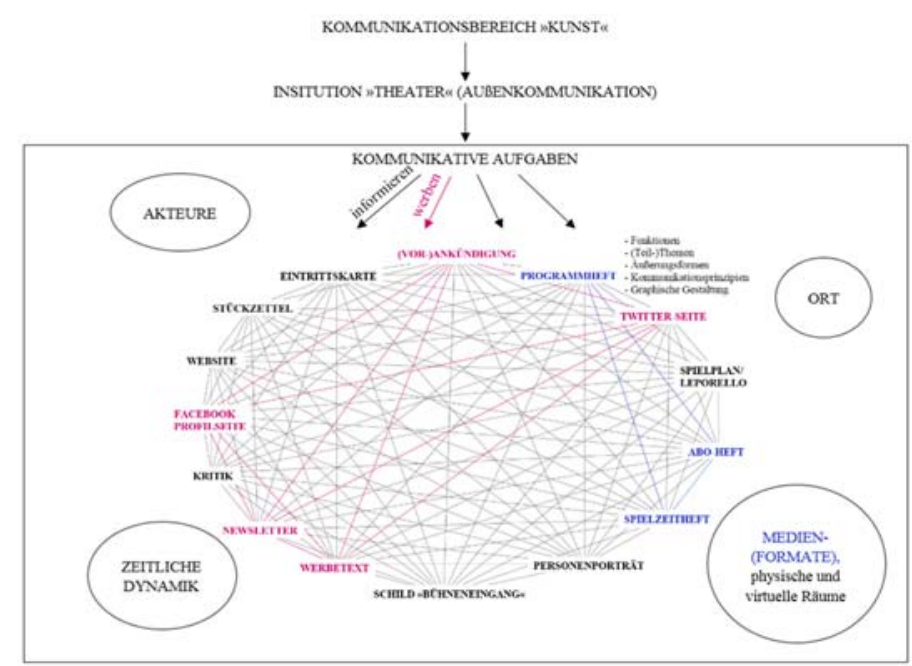

Grafik 1. Die Ökologie der Außenkommunikation von Theatern

Im Folgenden soll auf zwei wesentliche Aspekte dieser Ökologie, nämlich auf den physischen und den digitalen Kommunikationsraum (und innerhalb des physischen

\footnotetext{
${ }^{3}$ Unter ,empraktischen Kommunikaten“ (vgl. C. Domke 2014: 193f.) sind Kommunikationsangebote zu verstehen, die dem Rezipienten zu seinem Ziel verhelfen, wie z. B. Theaterkarten zu kaufen oder den Bühneneingang zu finden. Wie im Abschnitt 3 gezeigt wird, sind solche Kommunikationsangebote oft an einen bestimmten Ort gebunden bzw. gehören der sog. MesoKommunikation (C. Domke 2014) an.
} 
Raums insbesondere auf den Aspekt der Lokalität), eingegangen werden, um anschließend der Frage nachzugehen, welche Beziehungen sich zwischen den „physischen" und digitalen Kommunikationsangeboten feststellen lassen.

\section{Texte der Institution Theater im physischen und virtuellen Raum}

Texte und Kommunikationsangebote in der Außenkommunikation von Theatern finden in zwei Kommunikationsräumen statt: Im physischen und im virtuellen Raum. Bei der Analyse des physischen Kommunikationsraums der Institution Theater sollen in diesem Beitrag - im Gegensatz zu dem geplanten Forschungsprojekt, in welchem auch der Massenkommunikation angehörende Textsorten wie etwa Programmhefte oder Zeitungsankündigungen analysiert werden - vor allem der sogenannten „MesoKommunikation" (vgl. C. Domke 2014) angehörende Texte und Kommunikationsangebote im Umkreis von Theatern berücksichtigt werden. Diese Art von Kommunikation richtet sich „konträr zur zeitgleichen Ansprache von Massen über Massenmedien und zur singulären Adressierung in der Face-to-Face-Kommunikation [...] allein an die an einem Ort Anwesenden" (C. Domke 2014: 22). Dabei soll die Kategorie der „Lokalität“ (vgl. U. Fix 2008) nicht unberücksichtigt bleiben, denn im Rahmen der Meso-Kommunikation übernimmt der Ort der Texte eine bedeutungskonstitutive Rolle. Das heißt, dass die Orte, an denen sich Texte wie etwa Werbeplakate für Theateraufführungen, Spielpläne usw. befinden, das kommunikative Potential dieser Texte mitbestimmen und die Rezeption der Vorbeigehenden lenken. Viele „empraktische Kommunikate" (vgl. C. Domke 2014: 193f.) wie beispielsweise das Schild „Bühneneingang“, der Wegweiser zur Theaterkasse oder verschiedene Inschriften an Theaterhäusern sind sogar an das Theatergebäude gebunden, sie eröffnen ganz bestimmte Handlungsräume und wären an anderen Orten in ihrer spezifischen Funktion nicht verstehbar.

In den letzten Jahrzehnten wurden viele „klassische“ Texte durch digitale Kommunikationsangebote ergänzt oder sogar ersetzt. Dies ist auch in der kommunikativen Ökologie der Institution Theater der Fall. Im Folgenden sollen somit der Meso-Kommunikation angehörende, ,physische“ Texte und digitale (massenmediale) Kommunikationsangebote im Umkreis von Theaterinstitutionen gegenübergestellt werden. Dabei stellen sich u. a. folgende Fragen ${ }^{4}$ : Was sind die charakteristischen Merkmale der ortsgebundenen Texte im physischen Raum und was die der Texte im digitalen Raum der Theaterinstitutionen? Können die digitalen Texte, die aufgrund ihrer Virtualität immer und überall rezipiert werden, in irgendeiner Weise an die Kategorie der Lokalität gebunden werden? Inwiefern sind Texte in den beiden kommunikativen Räumen der Institution Theater miteinander verbunden?

Diese Fragen sollen im Beitrag anhand einer exemplarischen Analyse der Texte bzw. multimodalen Angebote aus dem Stadttheater Gießen, dem Nationaltheater Mannheim und dem Schauspiel Leipzig beantwortet werden.

${ }^{4}$ Die Forschungsfragen beziehen sich auf den Schwerpunkt der Sektion „Textlinguistik und Stilistik“ der im Jahr 2017 an der Universität Basel stattgefundenen Tagung der Gesellschaft für Angewandte Linguistik (GAL). Der Titel der Sektion lautete „Texte zwischen Lokalität und Virtualität". 


\subsection{Texte der Institution Theater im physischen Raum und ihre Lokalität}

\subsubsection{Nicht-ortsgebundene Kommunikation der Institution Theater}

Wenn man sich Theaterinstitutionen und ihre Umgebungen aus unmittelbarer Nähe anschaut, bemerkt man, dass diese - wie der öffentlich begehbare Raum schlechthin (vgl. C. Domke 2014: 20) - in großem Maße betextet werden. Dabei sind natürlich nicht alle Texte, die sich im Theater bzw. in seiner Nähe befinden, auch ortsgebunden. So sind in der Nähe von Theatergebäuden oft verschiedene Werbeplakate für Theateraufführungen oder Spielpläne vorzufinden. Während Werbeplakate die Funktion erfüllen, das Interesse der Vorbeigehenden für eine bestimmte Theateraufführung zu wecken und sie in ihrer Entscheidung für den Besuch einer Aufführung zu beeinflussen (und erst in einem zweiten Schritt über die Theateraufführung zu informieren), ist die Funktion der vor dem Theatergebäude ausgehängten Spielpläne, die potenziellen Theaterbesucher auf das Angebot des Theaters aufmerksam zu machen, vor allem aber über den Monatsplan/Wochenplan der Theateraufführungen zu informieren. Solche Texte wie Werbeplakate und Spielpläne, die vor dem Theatergebäude aushängen, sind zwar nicht indexikalisch in dem Sinne, dass sie nur an einem Ort interpretierbar sind - solchen Spielplänen können wir nämlich z. B. auch an Werbewänden verschiedener Universitätsgebäude oder in Bibliotheken begegnen, und Werbeplakaten für Theateraufführungen unter anderem auch in U-Bahnhöfen oder auf mobilen Objekten (wie etwa Bussen). Trotzdem sind solche nicht-indexikalischen Texte an manchen Orten passender als an anderen (vgl. P. Auer 2010: 279) - Werbetexte für Theateraufführungen und Spielpläne werden an Orten, die mit Kultur und Bildung verbunden sind, wahrscheinlich eher ihre Funktion erfüllen als beispielsweise auf einem Wochenmarkt oder einem Freibadgelände. Obwohl ortsungebunden, sind diese Texte also situationsbzw. kontextgebunden und lenken durch den Ort ihrer Publikation die Rezeption.

Unter dem Begriff „Lokalität“" werden in diesem Beitrag also sowohl Aspekte des Ortes verstanden, an welchen Texte gebunden sind (indexikalische öffentliche Zeichen), als auch des Ortes, an welchem Texte trotz ihrer Ortsungebundenheit eine bestimmte Bedeutung vermitteln (nicht-indexikalische öffentliche Zeichen). Im Folgenden soll die Aufmerksamkeit jedoch dem ersten Typ, den ortsgebundenen/ indexikalischen Texten im Umkreis der Institution Theater gewidmet werden.

\subsubsection{Ortsgebundene Kommunikation der Institution Theater: Funktionen, (Teil-)Themen, Formulierungsmuster, Textträger und Multimodalität}

Ausgehend von einer pragmatisch orientierten, d.h. einer funktionalen, handlungsbezogenen bzw. kommunikationsorientierten Texttheorie (vgl. K. Brinker 2005) werden Texte als „Werkzeuge der Kommunikation und als Mittel der Lösung kommunikativer Aufgaben“ (H.-Y. Cheng/ T. Gloning 2017: 14) aufgefasst. Sie sind von verschiedenen kommunikativen Aspekten der Textorganisation geprägt, die sich bei der Beschreibung von Texten und Kommunikationsangeboten im Umkreis von Theaterinstitutionen nutzen lassen. $\mathrm{Zu}$ solchen grundlegenden Parametern gehören u.a. die Funktionen, die Handlungsstruktur, Aspekte der Themenorganisation, typische sprachliche 
Mittel und andere semiotische Ressourcen, durch welche die kommunikativen Aufgaben realisiert werden. Eine wichtige Rolle bei der Beschreibung von Texten spielt außerdem die mediale Komponente, denn die technisch-physikalischen Gegebenheiten des Mediums bzw. des Textträgers prägen die Funktionen und das Bedeutungspotenzial von Texten mit. Im Folgenden sollen also einige wesentliche Charakteristika der ortsgebundenen Texte im physischen Raum der Institution Theater beschrieben werden, um festzustellen, zu welchen Zwecken, wie und worüber die Institution Theater mit vor Ort anwesenden Theaterbesuchern und am Theater Interessierten kommuniziert.

An das Theatergebäude und seine Umgebung gebundene Kommunikationsangebote erfüllen fünf grundlegende Funktionen, die in den folgenden sprachlichen Handlungen zum Ausdruck kommen: 1) benennen und charakterisieren, 2) über einen Ort informieren, 3) Angaben zum Gebrauch eines Ortes machen und bestimmte Handlungen eröffnen, 4) Wege weisen sowie 5) gedenken. Dabei können sich diese Funktionen auch überlappen.

1) Im Kommunikationsbereich „Theater“ werden vor allem die Theaterinstitutionen selbst benannt, wie etwa auf der Fahne mit dem Namen Nationaltheater Mannheim zu sehen ist (vgl. Abb. 1). Ein weiteres Beispiel für die Funktion der Benennung ist Haus der Karten des Stadttheaters Gießen (vgl. Abb. 2) hier wird das benannte Gebäude durch die Lokalangabe im Dürerhaus weiter charakterisiert. Solche Bezeichnungen im Kommunikationsbereich „Theater“" dienen allerdings nicht nur der Benennung an sich, vielmehr stehen sie im Dienst einer leichteren Orientierung, sie implizieren zum Teil aber auch schon Angaben über bestimmte Handlungen. So suggeriert die Inschrift Nationaltheater Mannheim (Abb. 1), dass man dort in Mannheim ins Theater gehen kann, und der Name Haus der Karten (Abb. 2), dass man dort Theaterkarten kaufen kann. Letztere implizite Angabe wird in Abb. 2 durch die Inschrift Theaterkasse + Tickets unterstützt.

2) Das Schild, das sich auf dem Steinzaun vor dem Eingang in das Stadttheater Gießen befindet und ebenfalls ortsgebunden ist (vgl. Abb. 3), informiert Vorbeigehende bzw. vor allem am Theater interessierte Touristen über das Stadttheater Gießen, seine Architektur und Geschichte. Darauf, dass die Informationen auf dem Schild wahrscheinlich in erster Linie für Stadtbesucher gedacht sind, weisen vor allem die nicht-sprachlichen Textelemente ${ }^{5}$ hin: Das rote Kästchen mit der Nummer 15 rechts neben der Überschrift Stadttheater suggeriert, dass es sich nur um eine der vielen Sehenswürdigkeiten in Gießen handelt; der Ausschnitt des Stadtplans rechts unten bestätigt dies und ordnet die einzelnen Sehenswürdigkeiten (vor allem das Stadttheater) in der Stadt ein.

3) Direkte Angaben zum Gebrauch eines Ortes zu machen ist nach P. Auer (2010: 292) „heute die wichtigste Funktion öffentlicher, ortsfester Schrift“. Hierbei kann sich in Theatergebäuden um einfache, eher knappe Funktionsbezeichnungen handeln, wie etwa im Fall der Schilder Theaterkasse, Abobüro (vgl. Abb.

\footnotetext{
${ }^{5}$ Zur graphischen Gestaltung der ortsgebundenen Texte siehe weiter unten.
} 
4) oder Bühneneingang (vgl. Abb. 5), es kommen aber nicht selten auch ausführlichere Angaben vor - beispielsweise darüber, welche Öffnungszeiten gelten. Die Funktion ,direkte Angaben zum Gebrauch eines Ortes machen bzw. bestimmte Handlungen eröffnen" erfüllt auch das Schild des Nationaltheaters Mannheim Abfahrt SIGNA-Shuttle ab der Bushaltestelle am Theatercafé (vgl. Abb. 6), das sich an der Haltestelle befindet, von der der Shuttlebus zur Aufführung Das Heuvolk des dänisch-österreichischen Künstler-Duos SIGNA abfährt. Dieses Schild erlangt durch das Zusammenspiel der Platzierung und der Wissensbestände der Rezipierenden (zum Wort SIGNA) Bedeutung. Ein weiteres Beispiel für die Funktion „Angaben zum Gebrauch eines Ortes machen“ sind im Kommunikationsbereich Theater verschiedene Schilder mit Hinweisen zum Gebrauch der Gästebücher.

4) Manche Kommunikationsangebote im Umfeld der Institution Theater erfüllen - wie viele andere ortsfeste Zeichen - die Funktion, Wege zu weisen. Solche Wegweiser verweisen oft auf entfernte Orte und enthalten einen Pfeil, der als distaler Pointer fungiert (vgl. P. Auer 2010: 278). Auch diese Texte können einfach oder komplex sein: Während z. B. das Schild des Mannheimer Nationaltheaters Lobby Werkhaus Studio (Abb. 7) knapper formuliert ist, handelt es sich im Fall des Wegweisers zur Theaterkasse des Stadttheaters Gießen (Abb. 8) um ein komplexeres Zeichen, das neben dem Wort Theaterkasse, dem Pfeil, der Anschrift sowie der Angabe darüber, wo sich die Kasse befindet (im Sparkassengebäude, rechts um das Theater), auch die Informationen zu den Öffnungszeiten der Kasse sowie einige allgemeine Angaben (Telefon- und Faxnummer sowie die E-Mail-Adresse) über das Theater enthält. Das Schild hat also eine doppelte Funktion: Zum einen geht es um das Wegweisen und zum anderen um das Informieren.

5) Um die Funktion des Gedenkens handelt es sich beispielsweise, wenn sich in der Nähe eines Theatergebäudes ein Denkmal eines verstorbenen Schriftstellers befindet. Diese Funktion erfüllen aber auch bestimmte Inschriften auf Theatergebäuden wie z. B. Ein Denkmal bürgerlichen Gemeinsinns auf dem Gebäude des Gießener Stadttheaters, die daran erinnern soll, dass am Ende des 19. Jahrhunderts die Spenden aus der Bevölkerung den Theaterbau ermöglicht haben.

Die oben beschriebenen übergeordneten Funktionen der ortsgebundenen Kommunikation im physischen Raum der Theaterinstitutionen (benennen, informieren, Angaben zum Gebrauch des Ortes machen, Wege weisen und gedenken) können mit verschiedenen thematischen Aspekten kombiniert werden. Dadurch ergeben sich Textbausteine mit Teilfunktionen (vgl. T. Gloning 2008: 65) wie, ,informieren über die Architektur und Geschichte des Schauspiels“, ,informieren über die Öffnungszeiten der Theaterkasse“, „Angaben zum Gebrauch der Theaterkasse geben“, „Angaben zum Gebrauch des Abobüros geben“, „Angaben zum Gebrauch der Bushaltestelle am Theatercafé geben“, „,wegweisen zur Theaterkasse“, „wegweisen zu verschiedenen Räumlichkeiten/Bühnen des Theaters“" (z. B. das Schild Lobby Werkhaus Studio - vgl. Abb. 7) usw. Im Fall der ortsgebundenen Kommunikate der Institution Theater lassen 
sich zwischen den einzelnen Handlungen außerdem typische Abfolgen beschreiben wie etwa ,"̈ber einen Ort informieren und dann Angaben zum Gebrauch dieses Ortes machen“ oder „zu einem Ort wegweisen und dann über diesen Ort informieren“ usw.

Was die Themenstruktur dieser empraktischen Texte anbelangt, so werden darin überwiegend die Gegenstände/Orte thematisiert, auf denen sich die Texte befinden bzw. auf die sie sich beziehen: die Theaterkasse, das Abobüro, der Bühneneingang, das Haus der Karten, die Bushaltestelle usw. Während es sich in den meisten Fällen um Kurztexte handelt, die oft aus einem Satz oder sogar nur aus einem Wort bestehen und daher eher wenig thematische Entfaltung aufweisen, sind in ausführlicheren ortsgebundenen Texten unterschiedliche Zusammenhänge zwischen einzelnen thematischen Elementen vorhanden, beispielsweise Fortführungszusammenhänge (z. B. zunächst das Wegweisen zur Theaterkasse und dann die Angaben zu den Öffnungszeiten - vgl. Abb. 8) oder Spezifisierungszusammenhänge (z. B. Thematisierung des Stadttheaters Gießen und seiner Geschichte und Architektur - vgl. Abb. 3).

Für die kommunikativen Aufgaben, die mit ortsgebundenen Texten der Institution Theater erledigt werden können, bestehen bestimmte mehr oder weniger standardisierte Vertextungsverfahren und Äußerungsformen. Laut P. Auer (2010: 288) hat Schrift im öffentlichen Raum „,normalerweise nicht viel Platz; sie soll aus der Distanz sichtbar sein". Zu den typischen Vertextungsverfahren gehören daher vor allem Formen der syntaktischen Verdichtung und Präsentationsökonomie. Dabei sind z. B. die modalen (deontischen) Infinitive wie etwa in Bitte Bügel drücken! zu erwähnen (vgl. ebd.), „die ihre pragmatische Kraft ohne alle Morphologie eindeutig [...] entwickeln können" (ebd., 289). Dazu gehören aber auch andere verblose bzw. nominalisierte Konstruktionen wie z. B. Eingang nur beim Pförtner (vgl. Abb. 9) oder Kurztexte, die nur aus einem Wort bestehen, wie etwa Bühneneingang (vgl. Abb. 5). Dass solche ortsgebundenen und grammatisch recht reduzierten Texte problemlos verstanden werden können, liegt laut P. Auer (2010: 289) zum einen an der sog. Form/FunktionKopplung - das heißt, dass bestimmte grammatische Konstruktionen (wie z. B. deontische Infinitive) mit bestimmten Funktionen (z. B. „Gebrauchsweisen vorschlagen/ verbieten") verbunden sind. Weil eine bestimmte Konstruktion mit einer bestimmten Funktion gekoppelt ist, kann auf weitere Angaben (z. B. zu Zeit, Person, Modus usw.) verzichtet werden. Zum anderen macht in bestimmten Situationen der Handlungskontext ,bereits so starke Vorgaben [...], dass eine sprachliche Explizierung verschiedener Situationsparameter nicht mehr nötig ist" (ebd.). Trotz aller empraktischen Einbindung setzt die Interpretation solcher ortsgebundenen Texte ein bestimmtes Allgemein- und vor allem spezifisches Sprach- bzw. Textsortenwissen sowie kulturelles Wissen voraus.

Die Auswahl der typischen sprachlichen Mittel (reduzierte Syntax, Einwortkonstruktionen etc.) ist an die technisch-physikalischen Gegebenheiten des Textträgers dieser Kommunikationsangebote angepasst. Im Interaktionsrahmen Theater wird im Hinblick auf Textträger vor allem durch Inschriften und Schilder kommuniziert. Während Inschriften dingfest sind (z. B. der Text Ein Denkmal bürgerlichen Gemeinsinns auf dem Hauptgebäude des Gießener Stadttheaters oder Haus der Karten auf dem 
Gebäude der Gießener Theaterkasse (vgl. Abb. 2)), können Schilder direkt oder indirekt mit dem Objekt verbunden sein, auf das sie sich beziehen (vgl. Abb. 3, 4, 5, 6, 7, 8, 9). $\mathrm{Zu}$ den Textträgern gehören außerdem auch Zettel, die eher temporär und normalerweise angeheftet sind. So wurde neben der Angabe zu den Öffnungszeiten des Nationaltheaters Mannheim beispielsweise ein Zettel angeheftet, der die potenziellen Käufer bzw. Theaterbesucher über die veränderten Öffnungszeiten am 29. Juni informiert (vgl. Abb. 10). In solchen Fällen, wo mehrere Schilder/ Inschriften/ Zettel nebeneinander stehen und sich inhaltlich aufeinander beziehen, kann von sog. Ensembles und Überschichtungen gesprochen werden. Im Fall der Abb. 10 handelt es sich um eine Überschichtung, da hier eine (zeitliche) Trennung zwischen dem früheren/primären Zeichen (also der Angabe zu den Öffnungszeiten) und dem späteren/sekundären Zeichen (dem Zettel mit der Information zur Änderung der Öffnungszeiten) besteht (vgl. ebd.: 287). Um ein Ensemble zweier Schilder handelt es sich in Abb. 9: Chorsaal/Orchesterprobensaal. Eingang nur beim Pförtner. Hier wurden vermutlich beide Schilder zugleich angebracht.

Um die übergeordneten Funktionen des Benennens, Informierens, Wegweisens usw. zu erfüllen, werden in ortsgebundenen Theater-Texten neben der Modalität „Sprache“ auch weitere Modalitäten bzw. semiotische Ressourcen verwendet - die ortsgebundenen Texte der Institution Theater sind hochgradig multimodal gestaltet ${ }^{6}$. Hierbei wird von der sog. „Theorie des multimodalen Handelns“ (H.J. Bucher 2010) ausgegangen, nach der verschiedene semiotische Ressourcen wie etwa Sprache, Bild, Typographie, Layout, Farbe usw. gewisse Zwecke erfüllen können. Was z. B. die Typographie der meso-kommunikativen Theater-Texte anbelangt, so ist die Schriftgröße der alleinstehenden Benennungen und der Überschriften (in längeren Texten) in den meisten behandelten Beispielen relativ groß; oft werden auch Großbuchstaben verwendet (vgl. z. B. Abb. 1, 2, 3, 4, 8, teilweise 9). Die Großbuchstaben haben die Funktion eines sprachlichen und typographischen Blickfangs und sind, wie schon erwähnt, aus der Ferne einfacher zu bemerken. Oft sind weitere typographische Besonderheiten wie etwa Fettdruck oder Hervorhebung durch Farbe vorhanden. Solche typographischen Hervorhebungen dienen einer besseren Orientierung der am Theater Interessierten im öffentlichen Raum.

Neben den sprachlichen und typographischen Ressourcen enthalten z. B. viele Schilder des Stadttheaters Gießen auch das Logo des Theaters, welchem eine Wiedererkennungsrolle zukommt (vgl. Abb. 2, 5 und 8). Darüber hinaus sind oft vor allem indexikalische Zeichen wie etwa Pfeile bzw. Pointer vorhanden, die Theaterbesuchern den Weg zu vom Hauptgebäude entfernten Spielorten und anderen wichtigen TheaterEinrichtungen zeigen (vgl. Abb. 7 und 8). Es kommen zudem auch andere Mittel, die Lokalität ausdrücken, vor, wie z. B. der Ausschnitt des Stadtplans von Gießen in Abb. 3. Dieser dient, wie bereits erwähnt, einer leichteren Orientierung der Passanten im öffentlichen Raum.

${ }^{6}$ Zur Multimodalität siehe z. B.G. Kress/ T. van Leeuwen 2006, K.O'Halloran 2004, H. Stöckl 2004a und 2004b u.a.m., J.A. Bateman 2008, A. Baldry et al. 2010, H.J. Bucher 2010 und 2011. 
Dabei sollen die oben beschriebenen Eigenschaften der ortsgebundenen Kommunikation der Institution Theater als Tendenzen und keinesfalls als obligatorische Merkmale verstanden werden. Die ortsgebundenen Texte lassen also eine gewisse Offenheit zu - ihre (Teil-)Themen, Formulierungsmuster, Textträger und graphische Gestaltung hängen immer von TextproduzentInnen und ihren Relevanzentscheidungen, vor allem aber von der jeweiligen Theaterinstitution, ihren Angeboten und der Funktion eines bestimmten Textes ab.

\subsection{Texte der Institution Theater im virtuellen Raum: Funktionen, (Teil-)Themen, Formulierungsmuster, Medium und Multimodalität}

Die Außenkommunikation von Theatern erfolgt heutzutage auch durch digitale Kommunikationsformen. Im Folgenden sollen anhand dreier solcher Kommunikationsangebote - der Websites, der Facebook-Profilseiten und der Twitter-Seiten verschiedener Theaterinstitutionen (Stadttheather Gießen, Schauspiel Leipzig und Nationaltheater Mannheim) - einige zentrale Eigenschaften der digitalen Kommunikation der Institution Theater bestimmt werden. Anschließend wird auf diejenigen Aspekte eingegangen, anhand welcher Texte und Kommunikationsangebote im physischen und virtuellen Raum der Institution Theater miteinander verbunden sind (vgl. Kapitel 4).

Digitale Kommunikationsangebote der Institution Theater und ihre Komponenten erfüllen verschiedene Funktionen: 1) über eine bestimmte Theaterinstitution und ihre Angebote informieren, 2) sich selbst (die Theaterinstitution) präsentieren und für sie und ihre Angebote werben, 3) mit den (potenziellen) Theaterbesuchern (und anderen an der Theaterinstitution Interessierten) in Kontakt treten, 4) Dienstleistungen anbieten bzw. Nutzerhandlungen ermöglichen. Auch hier kann zwischen den einzelnen Funktionen nicht immer eine klare Trennungslinie gezogen werden (z. B. wenn die Kontaktaufnahme mit den potenziellen Theaterbesuchern zu ihrer aktiven Beteiligung bzw. zu Nutzerhandlungen führt).

1) Eine der primären Funktionen von Theater-Websites und ihren Komponenten ist es, über die Theaterinstitution und ihre Angebote zu informieren. Nach K. Lehnen (2006: 199) sind Websites ,im Internet online verfügbare Hypertexte“, wobei für Hypertexte die Eigenschaft der Nicht-Linearität besonders charakteristisch ist (vgl. A. Storrer 2000: 227) ${ }^{7}$. So können sich beispielsweise die am Stadttheater Gießen Interessierten auf der Homepage des Theaters (vgl. Abb. 11) über die nächsten Premieren, nächsten Termine und „News“ informieren, durch das Anklicken einzelner Links bzw. Titel der Aufführungen (z. B. Peter und der Wolf) oder verschiedener Themenbereiche in der Navigationsleiste oben (Kalender, Spielzeit, Das sind wir, Info, Junges Theater, Ticketshop) können sie zu weiteren Textteilen bzw. Modulen und somit zu weiteren Informationen gelangen. Wegen der nicht-linearen Struktur von Hypertexten können die Website-Besucher selbst auswählen, welche Informationen

\footnotetext{
${ }^{7}$ Neben der Nicht-Linearität nennt A. Storrer (2000: 227) als weitere definitorische Merkmale von Hypertexten die Multimodalität und die Eigenschaft, dass Hypertexte computerverwaltete Texte sind.
} 
für sie besonders von Interesse sind. Eine ähnliche Funktion des Informierens (diese Funktion ist aber nicht die einzige!) haben auch Facebook-Profilseiten der Theaterinstitutionen, die (wie die meisten digitalen Kommunikationsangebote) ebenfalls über die Eigenschaft Hypertextualität verfügen. So wird man beispielsweise durch verschiedene (oft durch mehrere Modalitäten aufbereitete) Posts und Ankündigungen auf der Facebook-Wand des Schauspiels Leipzig fast täglich über die Neuigkeiten und Veranstaltungen des Schauspiels informiert, z. B: DIE KONFERENZ DER TIERE nach Erich Kästner ab heute im Zoo Leipzig. TOI**TOI**TOI \& PEACE an Roscha A. Säidow und Retrofuturisten! Karten gibt's ausschließlich im Zoo. Alle Termine hier: http://bit.ly/2oUeTsPA (vgl. Abb. 12). Durch das Anklicken verschiedener Links und „Buttons“ in der Navigationsleiste (links) kann man zudem zu weiterem Informationsmaterial gelangen, das ebenfalls aus sprachlichen Texten, Bildern und Videos besteht, also multimodal aufbereitet ist.

2) Eine weitere Funktion der digitalen Texte der Institution Theater ist die Funktion, Werbung zu betreiben (vgl. K. Marx/ G. Weidacher 2014: 197). Diese ist eng mit der Funktion der Selbstdarstellung (vgl. J. Androutsopoulos 2010: 428) verzahnt. Neben den schon erwähnten Posts und Ankündigungen, Fotos und Videos wird die Aufmerksamkeit der Besucher auf Profilseiten der Theater in sozialen Netzwerken nicht selten z. B. auch durch Bewertungen der Theaterbesucher geweckt. Durch solche Prosa-Bewertungen, die eine moderne Version der Theaterkritiken sind, aber im Gegensatz zu diesen überwiegend von „Laien“ geschrieben werden, kann das Interesse der Rezipienten für bestimmte Veranstaltungen geweckt werden und die Theaterinstitutionen bekommen dadurch mehrere Freunde (auf Facebook) bzw. Followers (auf Twitter), die im Idealfall zu Kunden bzw. Theaterbesuchern werden. Beispiele für solche (überwiegend positiven) Bewertungen finden sich u. a. auf der Facebook-Seite des Schauspiels Leipzig (vgl. Abb. 13).

3) Eine der wichtigsten Funktionen der digitalen Kommunikationsangebote der Institution Theater, vor allem ihrer sozialen Netzwerke, ist die Funktion der Kontaktaufnahme mit potenziellen Theaterbesuchern bzw. anderen an der Institution Interessierten. J. Androutsopoulos (2010: 429) spricht hierbei von der sog. Interaktionsfunktion von Sprache im Web 2.0, allgemeiner ist auch von der Eigenschaft der Dialogizität der Texte im Internet die Rede (vgl. K. Marx/ G. Weidacher 2014: 193). Dabei geht es nicht nur darum, dass Theaterinstitutionen durch Posts und Ankündigungen mit den Rezipienten Kontakt aufnehmen, sondern auch bzw. vor allem darum, dass die Internetnutzer auf diese Posts antworten, sie kommentieren, liken und teilen können ${ }^{8}$. Diese Funktion

\footnotetext{
${ }^{8}$ Mit J. Androutsopoulos (2010: 421) ist das erste Kennzeichen des Web 2.0 die sog. Partizipation. Das bedeutet, dass Inhalte im Web 2.0 von den Nutzern ,selbst beigesteuert - hochgeladen, kommentiert, mit anderen Inhalten verknupft usw.“ werden können. „Dadurch kann jeder durchschnittlich befähigte Nutzer, sebst wenn er nicht programmieren kann, viel leichter als bisher aktiv an der Informations- und Meinungsverbreitung teilnehmen - das Motto lautet: Jeder kann mitmachen“ (de.wikipedia.org 2009, zitiert nach J. Androutsopoulos 2010: 421).
} 
erfüllen neben den Posts und Ankündigungen auf Facebook-Seiten (Funktion der Kontaktaufnahme) also auch die oben erwähnten Bewertungen, Kommentare der Tweets und der Posts auf Facebook usw. (Interaktionsfunktion). So wurde der Post auf der Facebook-Seite des Stadttheaters Leipzig zur Theateraufführung Die Konferenz der Tiere beispielsweise 18 mal gelikt, 2 mal geteilt und 2 mal kommentiert (vgl. Abb. 12).

4) Neben der informierenden Funktion, der Funktion der Selbstdarstellung und Werbung sowie der Interaktionsfunktion ist es eine weitere wichtige Funktion der digitalen Texte im Kommunikationsbereich Theater, Dienstleistungen anzubieten bzw. Nutzerhandlungen zu eröffnen. Hierzu gehört beispielsweise der Kartenverkauf, der auf Websites durch das Anklicken einzelner Theateraufführungen (vgl. Abb. 11) bzw. des im Menü vorhandenen Themenbereiches Ticketshop (vgl. ebd.) erfolgt, auf sozialen Netzwerken aber normalerweise durch die Angabe eines Links angeregt wird, den die Nutzer anklicken können, wie etwa in: Alle Termine und Karten hier: [Link]. Solche Angaben in digitalen Kommunikationsangeboten enthalten oft sprachliche Ausdrücke, die eine sogenannte Organisationsfunktion erfüllen - in dieser Funktion trägt Sprache zusammen mit anderen semiotischen Mitteln „dazu bei, die Bildschirmoberfläche als einen kohärenten virtuellen Raum zu konstituieren, der Nutzerhandlungen ermöglicht und vorstrukturiert (J. Androutsopoulos 2010: 429). Die zentrale Leistung sprachlicher Einheiten ist hier ihre Doppelfunktion als Überschriften und Hyperlink-Indikatoren, mittels derer Nutzer-Aktivitäten ausgeführt werden" (ebd.). Wie weiter unten im Beitrag gezeigt wird, kann diese Organisationsfunktion der Sprache in digitalen Texten mit der Funktion „Angaben zum Gebrauch eines Ortes machen und bestimmte Handlungen eröffnen" der ortsgebundenen Texte der Institution Theater in Verbindung gebracht werden.

Den oben beschriebenen Hauptfunktionen der digitalen Texte im Theater ist auBerdem die Funktion der Unterhaltung untergeordnet. Das bedeutet, dass man auf eine interessante, innovative und unterhaltende Art und Weise über die Inhalte und Angebote der Theaterinstitution informiert, für sie wirbt usw. Darüber hinaus können die oben beschriebenen zentralen Funktionen der digitalen Texte - ähnlich wie die Funktionen der ortsgebundenen Texte der Institution Theater - in Teilfunktionen zergliedert und mit verschiedenen thematischen Aspekten kombiniert werden: „Über die Theaterinstitution und ihre Geschichte informieren“, „Über das Team/die Schauspieler/das Ensemble informieren“, „Über die Spielorte informieren“, „Über den Kartenverkauf informieren“, „Über die Öffnungszeiten der Theaterkasse informieren“, „Über die Theateraufführungen informieren“, „Über den Anfang/das Ende der Sommerpause informieren“, „Für die Theaterinstitution werben“, „Für Theateraufführungen/bestimmte Veranstaltungen werben“, „Internetnutzer auf bestimmte Veranstaltungen/Theateraufführungen/Events aufmerksam machen“, „Internetnutzer zu bestimmten Veranstaltungen/ Theateraufführungen/Events einladen“, „Nach der Meinung der Internetnutzer zu bestimmten Veranstaltungen fragen“, „Theaterkarten verkaufen“, „Die potenziellen Theaterbesucher zum Kauf des Abonnements anregen“ 
usw. Auch hier lassen sich zwischen den einzelnen Handlungen typische Abfolgen beschreiben wie etwa „Über eine bestimmte Theateraufführung informieren und dann zum Kartenverkauf anregen“" usw.

Im Hinblick auf die Themenstruktur kann gesagt werden, dass alle mit Theaterinstitutionen verbundenen Erscheinungen auch mögliche Gegenstände sind, die in digitalen Texten der Institution Theater thematisiert werden können: die Theaterinstitution selbst und ihre Geschichte, das Personal/ Team/ Schauspieler/ Dramaturge/ Regisseure, verschiedene Spielorte/ Bühnen, Theateraufführungen/ Veranstaltungen/Events, Spielplan/ Kalender, Spielzeiten, Karten, Theaterkasse, Abonnements, Publikationen usw. Zwischen den einzelnen thematischen Elementen können auch in digitalen Texten Zusammenhänge bestehen. Am häufigsten sind hierbei hierarchische Zusammenhänge, die auch visuell zum Ausdruck kommen (können) - so sind z. B. auf der Website des Stadttheaters Gießen dem übergeordneten thematischen Gesichtspunkt/ der Rubrik Kalender verschiedene Monate untergeordnet, diesen wiederum verschiedene Tage, und diesen Veranstaltungen/Aufführungen, die an den Tagen stattfinden. Wenn man die Veranstaltungen anklickt, gelangt man zu weiteren Informationen wie Stückinfo, Besetzung, Termine/ Karten, die thematisch dem übergeordneten Gesichtspunkt/der angeklickten Theateraufführung untergeordnet sind. Solche hierarchischen, aber auch andere thematische Zusammenhänge werden durch die Hypertextualität der digitalen Texte navigierbar gemacht.

Auch die digitalen Texte der Institution Theater weisen bestimmte sprachliche Eigenschaften auf, die als mehr oder weniger typisch bezeichnet werden können. Während in redaktionellen Texten auf Websites und in sozialen Netzwerken der Theaterinstitutionen die Standardsprache verwendet wird, kommen durch die außerinstitutionelle, nichtprofessionelle Produktion öffentlich zugänglicher Texte (z. B. in Kommentaren auf Facebook- und Twitterseiten der Schauspielhäuser) nicht selten mündlichkeitsnahe Schreibstile und Nonstandardvarietäten vor. Zu informellen schriftlichen Kommunikationsstilen gehört beispielsweise auch der expressive Gebrauch von Orthografie und Interpunktion; weil an den Diskussionen auf Vernetzungsplattformen jeder teilnehmen kann, sind nicht selten aber auch sprachliche Fehler vorhanden (z. B. Freue mich auf morgen, gehe mit meine Kinder noch mal - vgl. den ersten Kommentar in Abb. 12). Des Weiteren kommen auch in digitalen Texten der Institution Theater Vertextungsverfahren der syntaktischen Verdichtung und Präsentationsökonomie vor - diese Eigenschaft hängt zum einen mit der Nicht-Linearität der digitalen Texte zusammen (die Rezipienten haben also „keine Zeit“, ausführliche Texte zu lesen, deswegen müssen diese knapp formuliert werden) und zum anderen mit der Organisationsfunktion der Sprache im Internet (dazu gehören z. B. einzelne Themenbereiche in Navigationsleisten, die oft nur aus einem Wort bestehen - siehe Abschnitt 4). Zum Dritten kann man in manchen Texten in sozialen Netzwerken (wie etwa auf Twitter) nur eine begrenzte Zahl von Zeichen verwenden - die Tweets werden deshalb nicht selten durch Ellipsen bzw. reduzierte Syntax gekennzeichnet, was im folgenden Beleg gut zum Ausdruck kommt: Schauspiel Leipzig @Schauspiel_LE: AUSGEZEICHNET! In der Kritikerumfrage von @theatermagazin für unsere Arbeit der vergangenen Spielzeit: [Link] (vgl. Abb. 14). 
Während das Medium, durch das die digitalen Texte der Institution Theater verbreitet werden, natürlich das Internet ist, ist die graphische Gestaltung dieser Texte durch die Multimodalität gekennzeichnet - diese stellt ein zentrales Merkmal der Texte im Internet dar (vgl. J. Androutsopoulos 2010: 433 sowie K. Marx/ G. Weidacher 2014: 188). Neben den in den meisten visuellen Medien üblichen Sprache-BildKombinationen und typographischen Besonderheiten enthalten Websites, FacebookPosts und Tweets auch im Kommunikationsbereich Theater weitere Verbindungen aus Text, Ton und bewegtem Bild (vgl. Abb. 12 und 14). Während die Verwendung verschiedener typographischer Elemente, Farben und Layouts vor allem der Hervorhebung bestimmter Inhalte, einer besseren Übersichtlichkeit und somit einer leichteren Orientierung der Nutzer im virtuellen Raum dient, erfüllen Bilder und Videos in digitalen Theater-Texten die Funktionen, das im sprachlichen Teil Ausgedrückte zu ergänzen, neue Informationen zu liefern oder z. B. eine Szene aus der im sprachlichen Teil thematisierten Theateraufführung visuell zu präsentieren.

Um den Rahmen dieses Beitrags jedoch nicht zu sprengen, breche ich die Auseinandersetzung mit den digitalen Texten der Institution Theater an dieser Stelle ab. Im Folgenden sollen einige Aspekte besonders hervorgehoben werden, die der digitalen und der ortsgebundenen Kommunikation des Theaters gemeinsam sind.

\section{Schlussbemerkungen: Zum Zusammenhang zwischen ortsgebundenen und digitalen Texten in der Außenkommunikation von Theatern}

Im vorliegenden Beitrag wurde zunächst der Gegenstand des umfangreicheren Forschungsprojektes, die „kommunikative Ökologie“ der Institution Theater, vorgestellt, um anschließend zwei wichtige Komponenten der Ökologie in der Außenkommunikation von Theatern, nämlich Kommunikationsangebote im physischen und virtuellen Raum dieser Ökologie zu analysieren. Dabei wurden bei der Analyse des physischen Kommunikationsraums insbesondere ortsgebundene, ,empraktische“ Texte, die der sogenannten Meso-Kommunikation angehören (C. Domke 2014), unter die Lupe genommen. Diese wurden dann den digitalen (massenmedialen) Kommunikationsangeboten im Umkreis von Theaterinstitutionen gegenübergestellt.

Aus der exemplarischen Darstellung der Merkmale von ortsgebundenen und digitalen Texten der Institution Theater geht hervor, dass die beiden „Typen“ von Kommunikationsangeboten im Kommunikationsbereich Theater einige Unterschiede, aber auch viele Ähnlichkeiten aufweisen. Zwischen den beiden Kommunikationsräumen der behandelten Textsortenökologie bestehen also gewisse Zusammenhänge.

Im Hinblick auf die sowohl in ortsgebundenen als auch in digitalen Theater-Texten thematisierten Gegenstände wie etwa Kartenverkauf, verschiedene Spielorte/Bühnen oder andere Theater-Einrichtungen (z. B. Theatercafé, Theaterbus usw.) kann von gewissen thematischen Zusammenhängen zwischen den beiden Typen von Kommunikationsangeboten gesprochen werden.

Was den funktionalen Bereich der behandelten Texte angeht, so sind die beiden Typen von Texten z. B. durch die informierende Funktion miteinander verbunden. So können Informationen zu Öffnungszeiten der Theaterkasse sowohl auf der Theater- 
Website als auch auf dem an das Theatergebäude angebrachte Schild gefunden werden. Darüber hinaus wurde bereits erwähnt, dass die sog. Organisationsfunktion der digitalen Texte den Funktionen „Angaben zum Gebrauch eines Ortes machen und bestimmte Handlungen eröffnen" der ortsgebundenen Texte der Institution Theater ähnelt. Laut J. Androutsopoulos (2010: 429) bestehen gegenwärtige Webseiten ,zu einem nicht geringen Anteil aus organisierender Sprache“. Dazu werden verschiedene Themenbereiche in der Navigationsleiste (z. B. Kalender, Spielzeit, Das sind wir, Info, Junges Theater, Ticketshop - vgl. Abb. 11) gezählt, die den Internetnutzern/am Theater Interessierten eine leichtere „Orientierung“ auf den Websites ermöglichen. So bezeichnet beispielsweise der Begriff Kalender die Funktion dieses Themenbereichs in der Navigationsleiste - er suggeriert also, dass man durch das Anklicken dieses „Buttons“ zu weiteren Informationen über die Termine der Theateraufführungen gelangen kann. Über eine ähnliche Funktion verfügen die ortsgebundenen Funktionsbezeichnungen auf den Schildern Theaterkasse, Abobüro (vgl. Abb. 4) bzw. Bühneneingang (vgl. Abb. 5), die (indirekte) Angaben darüber machen, dass man dort Theaterkarten kaufen, ein Abonnement abschließen bzw. zur Bühne kommen kann. Diese digitalen und ortsgebundenen (Teil-)Texte sind sich nicht nur in ihrer Funktion, sondern auch in ihrer knappen Formulierungsweise ähnlich - sie bestehen nämlich überwiegend aus einfachen Nomina oder Verben, Nominal- bzw. Verbalphrasen. Während der Grund für die syntaktische Verdichtung in ortsgebundenen Texten darin liegt, dass sie nicht viel Platz haben und aus der Ferne sichtbar sein sollen (vgl. Abschnitt 3.1.2 dieses Beitrags), ist die syntaktische Reduktion in digitalen Texten für die Nicht-Linearität dieser Texte sowie die Organisationsdimension ihrer Sprache charakteristisch; die Textkohärenz wird (sowohl in den ortsgebundenen als auch in den digitalen Texten) daher in einer ,visual syntax“ (T. van Leeuwen 2004: 17) hergestellt, „der Typografie, Farbgebung und Layout angehören“ (J. Androutsopoulos 2010: 430). Eine weitere Gemeinsamkeit besteht also darin, dass sowohl die ortsgebundenen als auch die digitalen Texte im Kommunikationsbereich Theater multimodal sind. Im Fall der Nutzung ähnlicher sprachlicher Mittel und anderer semiotischer Ressourcen kann von formalen Beziehungen zwischen den beiden Typen von Kommunikationsangeboten gesprochen werden.

Eine weitere grundlegende Ähnlichkeit zwischen „lokalen“ und digitalen TheaterTexten ist ebenfalls im Bereich der Textfunktionen zu konstatieren, und zwar geht es um die Funktion ,Wege weisen“. Diese Funktion kann einerseits im konkreten Sinne verstanden werden - wie das bei Wegweisern (z. B. zur Theaterkasse, vgl. Abb. 8) der Fall ist. Andererseits kann der Begriff ,Wege weisen“ aber auch im metaphorischen Sinne verstanden und auf die Funktion der Hinweise auf unterschiedliche Verlinkungen in digitalen Texten übertragen werden. Während die ,richtigen“ Wegweiser normalerweise auf entfernte Orte verweisen und einen Pfeil enthalten, der als distaler Pointer fungiert (vgl. P. Auer 2010: 278), wird dieser Pfeil in Hinweisen auf Verlinkungen in digitalen Texten oft durch lokaldeiktische Ausdrücke wie hier oder unter ersetzt: Z. B.: Alle Termine hier: [...] oder Es gibt noch Karten unter [...]. Weil solche Texte, die anhand von sprachlichen oder nicht-sprachlichen Mitteln auf andere Texte/Orte verweisen, nicht linear bzw. nicht, ,abgeschlossen“ sind, verfügen sie über 
die Eigenschaft Hypertextualität. Im Fall der „klassischen“ (ortsgebundenen) Texte könnte man somit von einer Art nicht-computerverwalteter Hypertextualität sprechen - hier stellen nämlich die Pfeile „Links“ zwischen den Texten und den entfernten Orten (z. B. der Theaterkasse) dar. Da solche sprachlichen und nicht-sprachlichen „Wegweiser" einer besseren Orientierung im physischen wie auch im virtuellen Raum dienen und somit ihre Funktion, oft aber auch ihre Form den „lokalen“/ortsgebundenen und den digitalen Texten gemeinsam ist, kann im Fall der Außenkommunikation von Theatern von einem weiteren funktionalen Zusammenhang zwischen den „lokalen“ und den digitalen Texten gesprochen werden.

Die digitalen Texte der Institution Theater weisen also fraglos gewisse thematische, formale (hinsichtlich typischer sprachlicher und graphischer Gestaltung) und wenn man die Metaphorik des digitalen Raums voraussetzt - vor allem gewisse funktionale Relationen zu ortsgebundenen Texten im physischen Raum auf.

\section{Literaturverzeichnis}

Adamzik, K. (2000), Was ist pragmatisch orientierte Textsortenforschung? In: K. Adamzik (Hrsg.), Textsorten. Reflexionen und Analysen. Tübingen, 91-112.

Adamzik, K. (2011) Textsortennetze (in:) S. Habscheid (Hrsg.), Textsorten, Handlungsmuster, Oberflächen. Linguistische Typologien der Kommunikation. Berlin/ New York, 367-385.

Androutsopoulos, J. (2010), Multimodal - intertextuell - heteroglossisch: Sprach-Gestalten in "Web 2.0"-Umgebungen (in:) A. Deppermann/ A. Linke (Hrsg.), Sprache intermedial - Stimme und Schrift, Bild und Ton. Berlin/ New York, 419-445.

Auer, P. (2010), Sprachliche Landschaften. Die Strukturierung des öffentlichen Raums durch die geschriebene Sprache. In: A. Deppermann/ A. Linke (Hrsg.): Sprache intermedial - Stimme und Schrift, Bild und Ton. Berlin/ New York, 271-300.

Baldry, A./ P. Thibault/ J. Lemke (2010), Multimodal transcription and text analysis. A multimedia toolkit and coursebook. London.

Bateman, J. A. (2008), Multimodality and genre. A foundation for the systematic analysis of multimodal documents. Basingstoke/ Hampshire.

Brinker, K. (2005), Linguistische Textanalyse. Eine Einführung in Grundbegriffe und Methoden. Berlin.

Bucher, H. J. (2010), Multimodalität - eine Universalie des Medienwandels: Problemstellungen und Theorien der Multimodalitätsforschung. In: H. J. Bucher/ T. Gloning/ K. Lehnen (Hrsg), Neue Medien - neue Formate. Ausdifferenzierung und Konvergenz in der Medienkommunikation. Frankfurt am Main/ New York, 41-79.

Bucher, H. J. (2011), Multimodales Verstehen oder Rezeption als Interaktion. Theoretische und empirische Grundlagen einer systematischen Analyse der Multimodalität. In: H. Dieckmannshenke/ M. Klemm/ H. Stöckl (Hrsg.), Bildlinguistik. Theorien - Methoden - Fallbeispiele. Berlin, 123-156.

Cheng H.-Y./ T. Gloning (2017), Spielarten des Personenporträts in Zeitungen: Strukturen und Funktionen eines Textmusters. Gießen. 
Domke, C. (2014), Die Betextung des öffentlichen Raumes. Eine Studie zur Spezifik von Meso-Kommunikation am Beispiel von Bahnhöfen, Innenstädten und Flughäfen. Heidelberg.

Fix, U. (2008), Nichtsprachliches als Textfaktor: Medialität, Materialität, Lokalität. In: „Zeitschrift für germanistische Linguistik“, 36.3, 343-354.

Gloning, T. (2008), "Man schlürft Schauspielkunst...". Spielarten der Theaterkritik. In: L. Hagestedt (Hrsg.), Literatur als Lust. Begegnungen zwischen Poesie und Wissenschaft. Festschrift für Thomas Anz. München, 59-86.

Janich, N. (2009), Zur Analyse von Textsorten-in-Vernetzung. Eine Modelldiskussion an einem Fallbeispiel aus der Unternehmenskommunikation. LAUD-Paper 734, Essen.

Klein, J. (1991), Politische Textsorten. In: K. Brinker (Hrsg.), Aspekte der Textlinguistik. Olms.

Klein, J. (2000), Intertextualität, Gestaltungsmodus, Texthandlungsmuster. Drei vernachlässigte Kategorien der Textsortenforschung - exemplifiziert an politischen und medialen Textsorten. In: K. Adamzik (Hrsg.), Textsorten. Reflexionen und Analysen. Tübingen, 31-44.

Kress, G./ T. van Leeuwen (2006), Reading images. The grammar of visual design. London.

Lehnen, K. (2006), Hypertext - kommunikative Anforderungen am Beispiel von Websites. In: P. Schlobinski (Hrsg.), Von „hdl“ bis ,cul8r“. Sprache und Kommunikation in den neuen Medien. Mannheim, 197-209.

Luckmann, T. (1986), Grundformen der gesellschaftlichen Vermittlung des Wissens: Kommunikative Gattungen.(in:) F. Niedhart/ M.R. Lepsius/ J. Weiss (Hrsg.), Kultur und Gesellschaft. René König, dem Begründer der Sonderhefte, zum 80. Geburtstag gewidmet. Opladen, 191-211.

Luckmann, T. (1997), Allgemeine Überlegungen zu kommunikativen Gattungen. In: B. Frank (Hrsg.), Gattungen mittelalterlicher Schriftlichkeit. Tübingen, 11-18.

Marx, K./ G. Weidacher (2014), Internetlinguistik. Ein Lehr- und Arbeitsbuch. Tübingen.

O’Halloran, K. (Hrsg.) (2004), Multimodal discourse analysis. Systemic functional perspectives. London.

Storrer, A. (2000), Was ist "hyper" am Hypertext? (in:) W. Kallmeyer (Hrsg.), Sprache und neue Medien. Berlin/New York, 222-249.

Stöckl, H. (2004a), Die Sprache im Bild - das Bild in der Sprache. Berlin/ New York.

Stöckl, H. (2004b), Typographie: Gewand und Körper des Textes - Linguistische Überlegungen zu typographischer Gestaltung. In: „Zeitschrift für angewandte Linguistik“ 41, 5-48.

Škerlavaj, T. (2018), Die Ökologie von Texttypen und multimodalen Angeboten in der Außenkommunikation von Theatern. Linguistische Grundlagen und Fallstudie. Gießen.

van Leeuwen, T. (2004), Ten reasons why linguists should pay attention to visual communication. In: P. LeVine/ R. Scollon (Hrsg), Discourse and technology: Multimodal discourse analysis. Washington, D.C., 7-19. 


\section{Anhang}
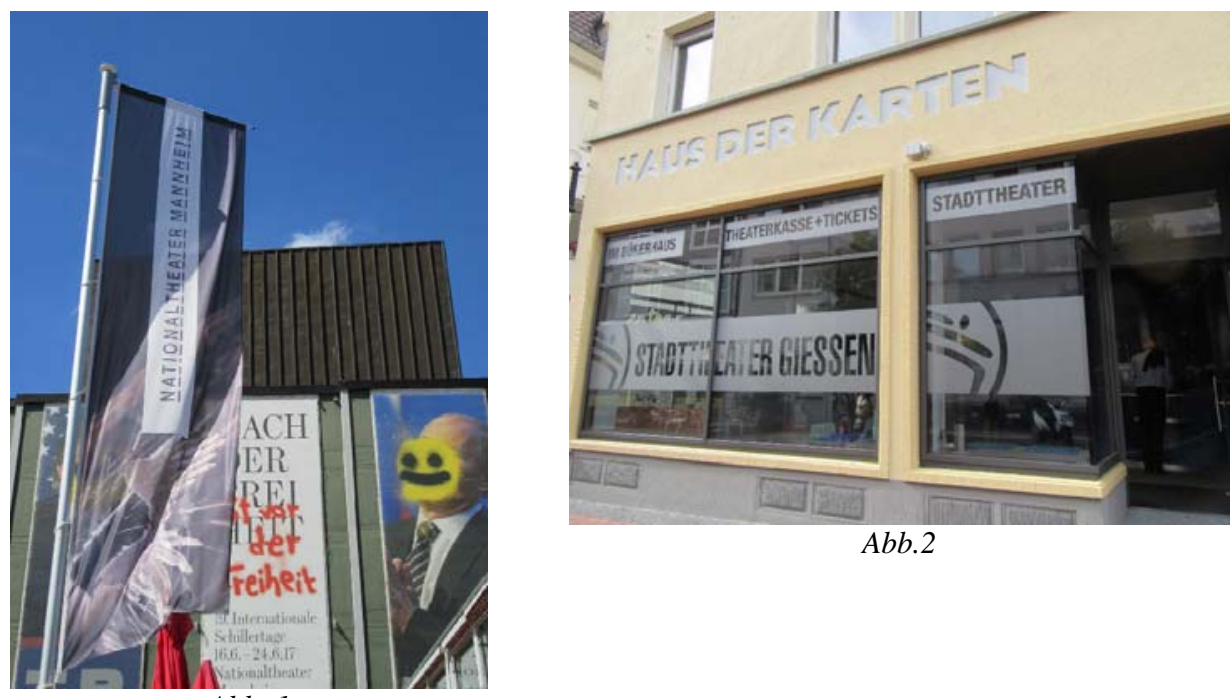

Abb.2

Abb. 1
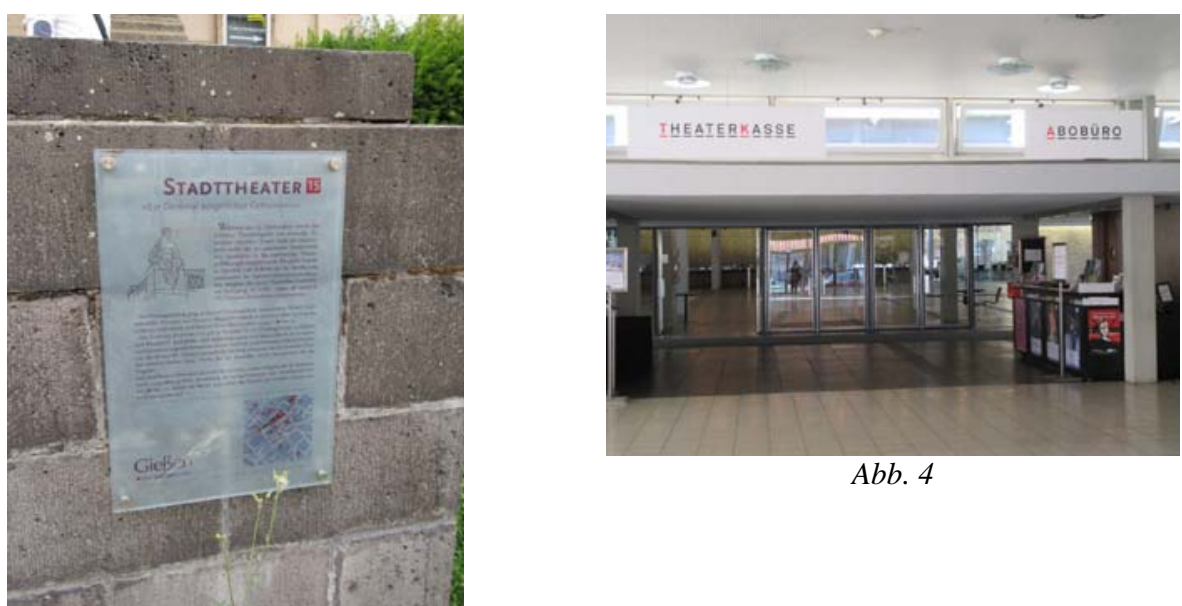

Abb. 4

Abb. 3 


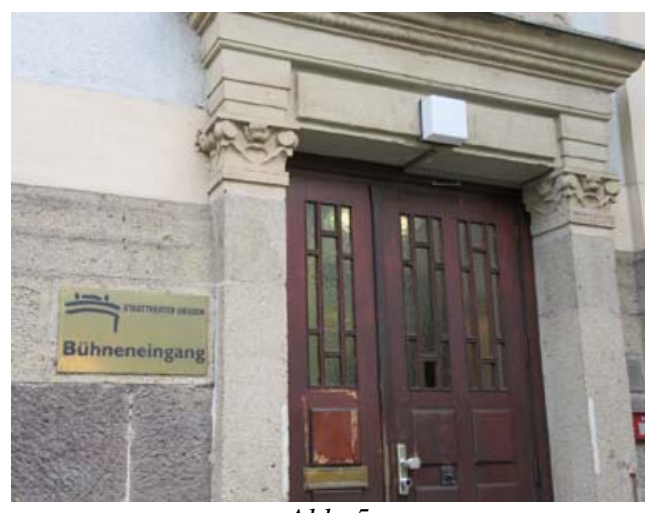

Abb. 5

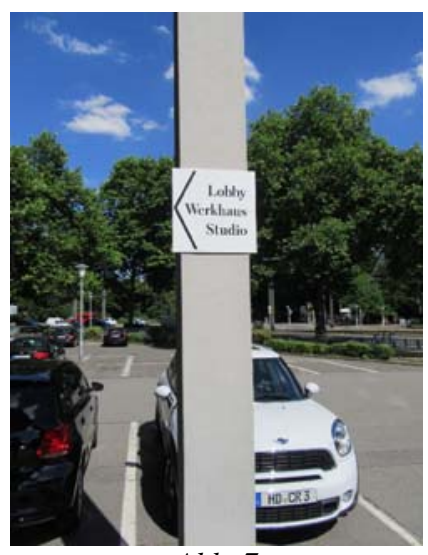

Abb. 7

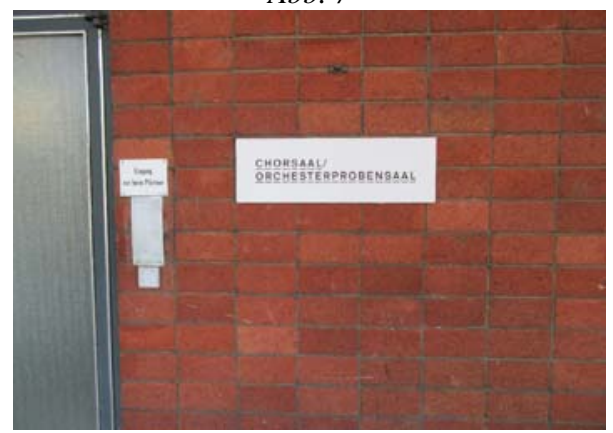

Abb. 9

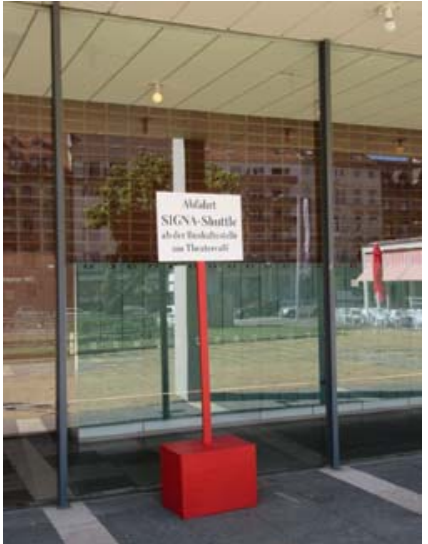

Abb. 6

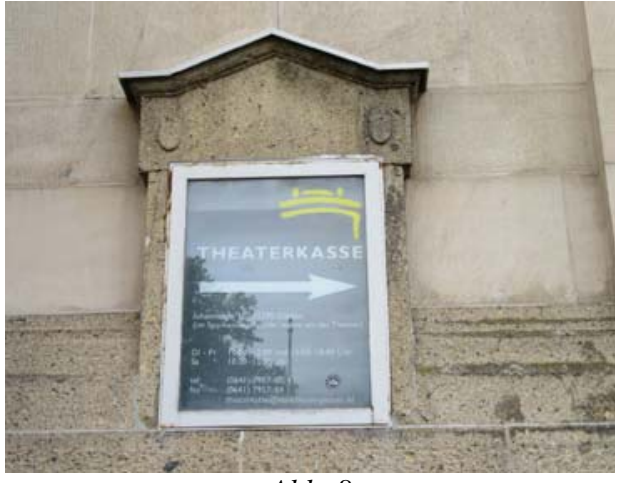

Abb. 8

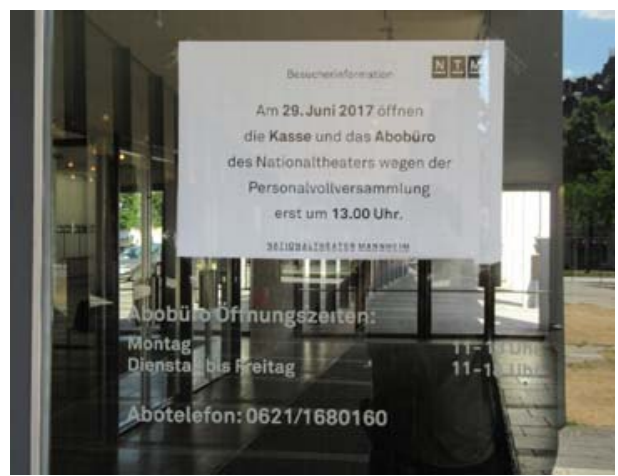

Abb. 10 

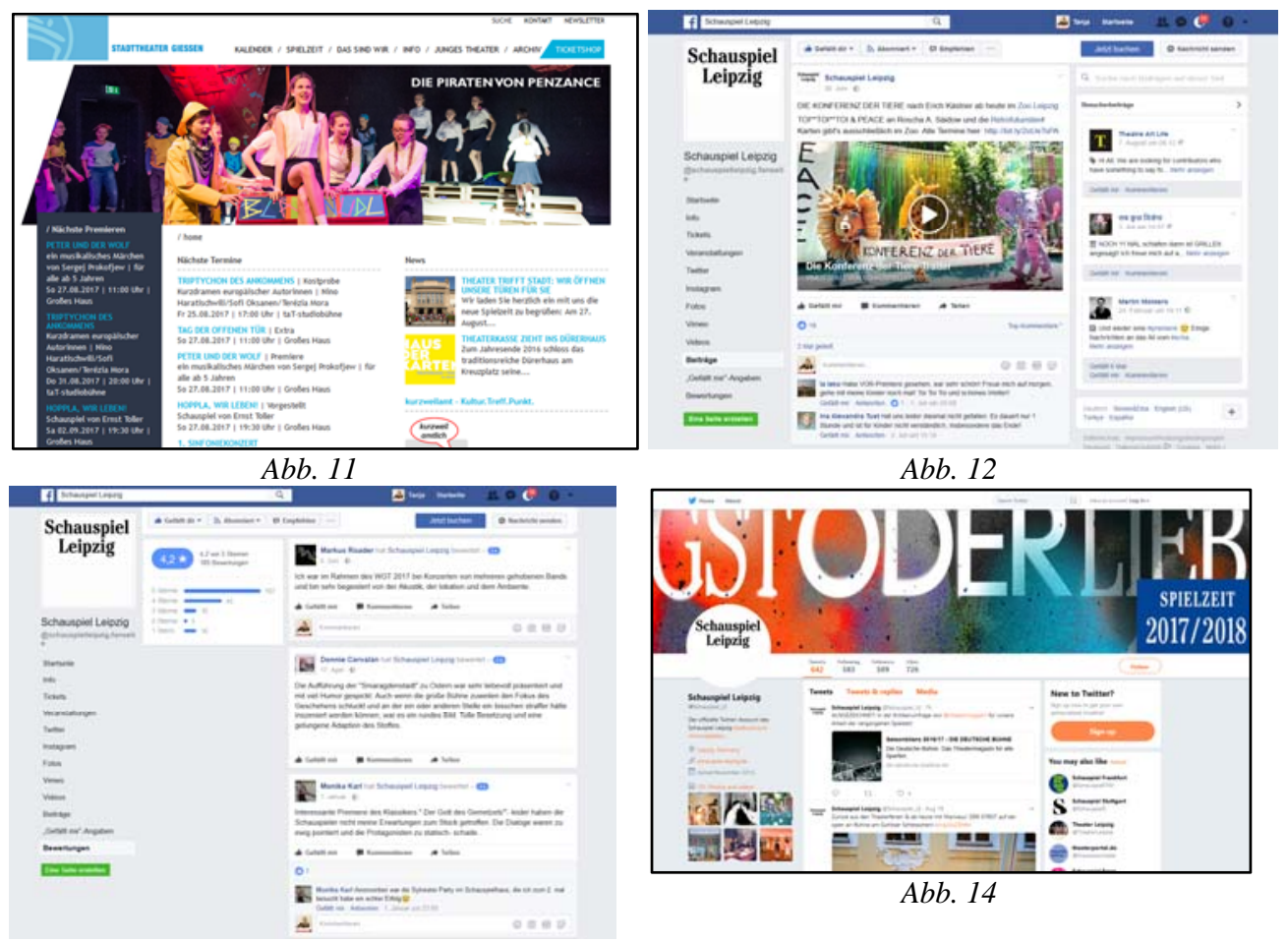

$A b b .13$ 